\title{
Hepatic oleate regulates liver stress response partially through PGC- 1a during high-carbohydrate feeding
}

Xueqing Liu ${ }^{1, \dagger}$, Maggie S. Burhans ${ }^{2, \dagger}$, Matthew T. Flowers ${ }^{1}$, James M. Ntambi ${ }^{1,2, \star}$

Departments of ${ }^{1}$ Biochemistry and ${ }^{2}$ Nutritional Sciences, University of WisconsinMadison, 433 Babcock Drive, Madison, WI 53706

${ }^{\#}$ These authors contributed equally as joint first authors

${ }^{*}$ Corresponding author. Address: Department of Biochemistry, University of WisconsinMadison, 433 Babcock Drive, Madison, WI 53706, USA. Tel.: +1 6082399074.

E-mail address: ntambi@biochem.wisc.edu (J. M. Ntambi)

Abbreviations: ER, endoplasmic reticulum; TG, triglycerides; CE, cholesterol esters; PL, phospholipids; FFA, free fatty acids; SFA, saturated fatty acids; MUFA, monounsaturated fatty acids; SCD, stearoyl-CoA desaturase; GKO, global knockout; HSVLF, high-sucrose very low-fat; LKO, liver-specific knockout; GLS5, GKO liverspecific SCD5; GLS3, GKO liver-specific Scd3; HC, high sucrose, high-carbohydrate; WT, wild-type; TLC, thin layer chromatography; GLC, gas liquid chromatography; ALT, alanine aminotransferase; UPR, unfolded protein response; DLKO, double liver knockout; ROS, reactive oxygen species 
Keywords: ER stress; Inflammation; Oleate; PGC-1a; Stearoyl-CoA desaturase

\section{Abstract [1]}

Background \& Aims: High-carbohydrate diets contribute to the development of liver stress and fatty liver disease. While saturated fatty acids are known to induce liver stress, the role of monounsaturated fatty acids (MUFA), synthesized by the stearoylCoA desaturase (SCD) family of enzymes, in regulation of liver function during lipogenic dietary conditions remains largely unknown. The major products of SCD-catalyzed reactions are oleate $(18: 1 n-9)$ and palmitoleate $(16: 1 n-7)$.

Methods: We generated mouse models with restricted exogenous MUFA supply and reduced endogenous MUFA synthesis, in which SCD1 global knockout (GKO) or liverspecific knockout (LKO) mice were fed a lipogenic high-sucrose very low-fat (HSVLF) or high-carbohydrate $(\mathrm{HC})$ diet. In a gain-of-function context, we introduced liver-specific expression of either human $S C D 5$, which synthesizes $18: 1 \mathrm{n}-9$, or mouse $S c d 3$, which synthesizes 16:1n-7, into SCD1 GKO mice and fed the HSVLF diet.

Results: Lipogenic high-carbohydrate diets induced hepatic endoplasmic reticulum (ER) stress and inflammation in SCD1 GKO and LKO mice. Dietary supplementation with 18:1n-9, but not 18:0, prevented the HSVLF diet-induced hepatic ER stress and inflammation in SCD1 LKO mice, while hepatic SCD5, but not Scd3, expression reduced the ER stress and inflammation in GKO mice. Additional experiments revealed liver-specific deletion of the transcriptional coactivator PGC-1 $\alpha$ reduced hepatic inflammatory and ER stress response gene expression in SCD1 LKO mice.

Conclusions: Our results demonstrate an indispensable role of hepatic oleate in protection against lipogenic diet-induced hepatic injury, and PGC-1a potentiates the ER 
stress response under conditions of restricted dietary oleate coupled to reduced capacity of endogenous hepatic oleate synthesis.

Lay summary: Susceptibility to metabolic dysfunction is influenced by genetic and environmental factors. In this study we show that modulation of two genes regulates the liver response, including ER stress and inflammation, to a high-carbohydrate low-fat diet. We reveal that hepatic availability of oleate, a monounsaturated fatty acid, is important for maintenance of liver health. 


\section{Introduction [1]}

The prevalence of obesity and associated metabolic diseases is a global epidemic. The etiology of these disorders is multifactorial and the nutritional environment is one such contributing factor (1). High-carbohydrate diets, especially those rich in sugars, promote lipid synthesis through induction of the de novo lipogenesis program, which is associated with unfavorable metabolic conditions such as hyperlipidemia and nonalcoholic fatty liver disease (2). Fatty acids and lipids are commonly implicated in mediating inflammation and endoplasmic reticulum (ER) stress (3-6) resulting in metabolic disturbances such as insulin resistance (IR) and nonalcoholic fatty liver disease (NAFLD) (7), and genetic and high-fat diet fed mouse models of obesity have been shown to develop ER stress (8). Additionally, the transcriptional coactivator peroxisome proliferator-activated receptor gamma, coactivator 1 alpha (Ppagrc1a; PGC-1a), which is a master regulator of mitochondrial biogenesis and lipid metabolism, has been linked to mediating ER stress in skeletal muscle in response to exercise (9). However, its interplay with various fatty acid species in regulation of ER stress in response to other stimuli has not been reported.

Major tissue lipids such as triglycerides (TG), cholesterol esters (CE), phospholipids ( $\mathrm{PL}$ ), wax esters and nonesterified free fatty acids (FFA) possess essential biological functions including maintenance of membrane integrity, cellular signaling regulation, and energy storage, among others. Synthesis of these lipids requires saturated fatty acids (SFA) and unsaturated fatty acids, with monounsaturated fatty acids (MUFA) being particularly essential $(10,11)$. MUFA are synthesized endogenously from SFA by the stearoyl-CoA desaturase (SCD) family of enzymes, and 
the major products of SCD-catalyzed reactions are oleate $(18: 1 n-9)$ and palmitoleate $(16: 1 n-7)$. There are four known isoforms of SCD in mice (SCD1-4) and two known isoforms in humans (SCD1 and 5). Despite high dietary abundance of 18:1n-9, the expression of the ubiquitously expressed isoform SCD1 is highly regulated by developmental, dietary, hormonal, and environmental factors. Because of the involvement of MUFA in the regulation of diverse processes including signal transduction, cell differentiation and neuronal development, SCD1 is regarded as an important enzyme in the regulation of normal and patho-physiological processes (1214).

While SFA are known to induce lipotoxicity, insulin resistance, liver stress and inflammation $(15,16)$, the specific role of hepatic MUFA in regulation of liver function has not been thoroughly studied. We previously demonstrated that hepatic stress and inflammation are highly induced in SCD1 global knockout mice (GKO) fed a lipogenic high-sucrose very low-fat (HSVLF) diet despite dramatic protection against hepatic steatosis and adiposity induced by this high-carbohydrate diet (17). To investigate the role specifically of hepatic MUFA in protection against high-carbohydrate diet-induced liver stress and inflammation, we utilized a model in which SCD1 liver knockout mice (LKO) were fed the HSVLF diet such that the exogenous MUFA supply and the endogenous capacity to induce MUFA synthesis were restricted. We demonstrate that liver-specific SCD1 deficiency is sufficient to induce hepatic ER stress and inflammation despite protection against diet-induced hepatic steatosis (18). Mechanistically, using liver-specific transgenic desaturase mice in the SCD1 GKO background we demonstrate differential effects of endogenously synthesized hepatic oleate and 
palmitoleate, two MUFA species, in regulation of lipogenic diet-induced stress and inflammation. We also reveal a role for PGC-1a in mediating hepatic ER stress under high-carbohydrate feeding. Taken together, these results reveal that hepatic oleate, either through exogenous dietary supply or endogenous de novo synthesis, is essential in the prevention or resolution of hepatic stress and inflammation induced by a lipogenic diet. 


\section{Materials and methods [1]}

\section{Animals and diets [2]}

All mice were of the C57BL/6 background. Scd1 $1^{\text {loxlox }}$ (Lox) mice and Scd1 $1^{\text {loxllox; }}$ Albumin Cre/+ (SCD1 LKO) mice were generated previously (18). For studies on the de novo synthesis of oleate and palmitoleate, two liver-specific transgenic lines were generated by cloning either the human $S C D 5$ or mouse $S c d 3$ cDNA sequence into the pLiv.LE6 vector construct (a gift from John Taylor, Gladstone Institute). The SCD5 and Scd3 transgenes were crossed into the SCD1 global knockout (GKO; Scd1 $1^{-1-}$ ) background to produce GKO liver-specific SCD5 (GLS5) and Scd3 (GLS3) transgenic mice, as previously described (19). To study the role of PGC-1a in SCD1 LKO mice, we crossed Ppargc1a ${ }^{\text {loxlox }}$ mice (a gift from Dr. Bruce Spiegelman) with Scd1 10xlox mice to generate Ppargc1 $a^{\text {loxl+t}} ; S c d 1^{|\mathrm{lox}|+}$ mice, which were then bred to generate Ppargc1 $\mathrm{a}^{\text {lox/lox; }}$ Scd1 10xllox mice. We then bred in the Cre recombinase allele, driven by the albumin promoter, to generate Ppargc1a $a^{\text {loxlox; }}$ Scd1 10x/lox; Albumin Cre/+ mice, in which both PGC$1 \alpha$ and SCD1 are deleted only from the liver (double liver knockout mice, DLKO).

\section{Statistical analysis [2]}

Data are expressed as mean \pm SEM. Statistical analysis with three or more groups was done using one-way ANOVA with Tukey's post hoc test, and the difference between two groups was tested by two-tailed, unpaired Student's $t$ test. Significance was considered when $p<0.05$.

For detailed experimental methods please see supplementary information 
Results [1]

Hepatic MUFA levels are reduced in SCD1 LKO mice fed a high-sucrose very lowfat diet [2]

Lox and SCD1 LKO mice were fed a standard chow diet or a lipogenic, HSVLF diet for 10 days to study the role of hepatic MUFA on liver function, including stress and inflammation in the context of a highly lipogenic dietary regimen. Among chow-fed Lox and SCD1 LKO mice there were no differences in the fatty acid composition of liver or plasma fatty acids (Fig. 1A-D). In HSVLF-fed mice however, the levels of oleate and palmitoleate, the major products of the SCD1 reaction, were lower in hepatic TG, FFA and CE fractions in SCD1 LKO as compared to Lox mice (Fig. 1A-C). Palmitate and stearate levels were not different in TG and FFA (Fig. 1A, B) but were significantly higher in the CE fraction in SCD1 LKO mice (Fig. 1C), likely due to a switch to SFA as substrates for cholesterol esterification to reduce the accumulation of free cholesterol. In plasma FFA, nonesterified oleate was significantly reduced in SCD1 LKO mice but there were no differences in SFA (Fig. 1D). Plasma albumin levels exhibited no genotypic difference (Supplementary Fig. 1), suggesting that any reductions in plasma fatty acids were not due to differences in fatty acid transport capacity. Overall, these data confirm that HSVLF-fed SCD1 LKO mice provide a model in which to study restricted hepatic MUFA availability, with dietary and endogenous supplies of MUFA to the liver significantly reduced in the absence of changes in SFA levels.

HSVLF-fed SCD1 LKO mice maintain normal MUFA levels in adipose tissue [2]

We next analyzed epididymal white adipose tissue TG fatty acids. There were no significant genotypic differences in either MUFA or SFA under chow or HSVLF dietary 
conditions (Fig. 2A). Additionally, hepatic SCD1 deficiency did not alter Scd1 mRNA or SCD1 protein levels in this tissue during HSVLF dietary feeding (Fig. 2B, C). The relative mRNA levels of other lipogenic genes such as Srebf1, Acaca and Fasn were also unchanged (Fig. 2B), suggesting that de novo fatty acid synthesis in adipose tissue of SCD1 LKO mice was not altered. Chow-fed Lox and SCD1 LKO mice also did not show differences in white adipose tissue lipogenic gene expression (data not shown). Thus, alterations in tissue fatty acid levels due to liver-specific SCD1 deficiency during a lipogenic diet are not extended to white adipose, and despite intact SCD1 expression in extrahepatic tissues, liver MUFA composition was not normalized.

\section{SCD1 LKO mice fed HSVLF exhibit liver injury and inflammation [2]}

We previously reported that SCD1 GKO mice develop liver dysfunction with stress and inflammation when fed the lipogenic HSVLF diet for 10 days (17). However, whether these phenotypes are specifically due to impaired hepatic MUFA synthesis is unknown. To address this issue, we assessed markers of stress and inflammation in HSVLF-fed SCD1 LKO mice. Plasma alanine aminotransferase (ALT) activity, a marker for liver damage, was increased nearly 20 -fold in SCD1 LKO mice relative to Lox but was not altered in chow-fed SCD1 LKO mice (Fig. 3A). Necrotic foci were also evident in liver sections of SCD1 LKO mice but not in Lox or chow-fed SCD1 LKO mice (Fig. 3B). In parallel with liver damage, HSVLF-fed SCD1 LKO mice had significantly higher expression levels of proinflammatory genes including Tnfa, Icam1 and Cc/2 (Mcp1) as compared to Lox mice (Fig. 3C). Additionally, hepatic expression of the macrophage markers Emr1 and Cd68 were approximately $\sim 3$ and $\sim 12$ fold higher, respectively, in SCD1 LKO mice (Fig. 3D). Liver inflammation in SCD1 LKO mice was consistent with a 
higher ratio of wet to dry liver weight, indicative of hepatic edema and liver inflammation (Fig. 3E). In a separate group of male mice we fed a high-sucrose high-carbohydrate $(\mathrm{HC})$ diet (12\% fat), more typical of diets consumed by humans, for 10 days and assessed the impact on gene expression markers of liver inflammation (Fig. 3F). Hepatic expression of Cc/2, Icam1 and Cd68 were significantly higher in LKO mice compared to Lox and a trend towards upregulated expression of Tnfa $(p<0.1)$ was detected.

Despite the presence of hepatic inflammation, systemic insulin sensitivity was not impaired but rather enhanced in HSVLF-fed SCD1 LKO mice (Supplementary Fig. 2) based on an insulin tolerance test. Also, 10 day HSVLF feeding did not induce inflammation in epididymal white adipose tissue, as $\mathrm{Ccl} 2$ expression and adipose tissue histology revealed no signs of inflammation in SCD1 LKO compared to Lox mice (Supplementary Fig. 3).

We also questioned whether the dramatic induction of the diet-induced liver inflammation in 10 day HSVLF-fed SCD1 LKO mice would be blunted upon longer-term feeding, during which feedback and compensatory response could readily occur. After 10 weeks of HSVLF feeding, plasma ALT activity remained significantly increased (Fig. 3G) and hepatic necrotic foci were evident (Fig. 3H) in SCD1 LKO but not Lox mice. However, the ALT activity in SCD1 LKO was approximately $50 \%$ of that in the short-term HSVLF-fed mice, suggesting that peripheral supply of fatty acids to the liver or resolution of the stress and inflammation through adaptation under the context of longterm feeding partially blunted the hepatic stress response. Taken together, these studies 
indicate that hepatic SCD1 is required for the maintenance of normal liver function under high-carbohydrate feeding when exogenous MUFA are restricted.

Dietary oleate supplementation rescues liver injury and inflammation in SCD1 LKO mice [2]

We then fed Lox and SCD1 LKO mice the HSVLF diet supplemented with either oleate or stearate, at a level we have previously shown to be absorbed (20), for 10 days to determine specifically whether decreased hepatic oleate was responsible for the HSVLF-induced liver injury. Oleate supplementation significantly increased hepatic TG and FFA oleate levels in SCD1 LKO mice (Supplementary Fig. 4). Dietary supplementation with oleate prevented the onset of HSVLF-induced liver inflammation, elevated plasma ALT activity, and development of liver necrotic foci in SCD1 LKO mice (Fig. 3A-D). In contrast, dietary supplementation of stearate did not prevent HSVLFinduced liver dysfunction and inflammation (Fig. 3). These results indicate that under lipogenic dietary conditions it is decreased hepatic MUFA, and not general hepatic fatty acid limitation, that leads to liver injury and inflammation.

Hepatic ER stress, but not mitochondrial stress, is induced in SCD1 LKO mice fed a lipogenic high-carbohydrate diet [2]

ER and mitochondria are regarded as major cellular stress response mediators (21-23). We questioned whether these organelles exhibited stress coincident with the HSVLF diet-induced hepatic inflammation. We assessed markers of the unfolded protein response (UPR), which is induced when ER proteins do not properly fold and initiates specific signaling cascades that ultimately lead to a reduction in the level of misfolded proteins (4). Upon activation of the UPR, protein kinase RNA-like ER kinase 
(PERK) phosphorylates eukaryotic translation-initiation factor $\alpha$ (elF2 $\alpha)$, which results in reduced general protein synthesis. Immunoblot analysis revealed that hepatic P-elF2 $\alpha$ was increased in HSVLF-fed SCD1 LKO mice (Fig. 4A). An increased level of spliced Xbox binding protein-1 (Xbp1) mRNA, which encodes for an active and potent transcription factor that cooperates with activating transcription factor $6 \alpha$ (ATF6a) to increase expression of stress-responsive genes, indicates activation of the IRE1 $\alpha$ pathway (4). The relative mRNA level of spliced $X b p 1$, but not total, was significantly elevated by HSVLF feeding in SCD1 LKO mice (Fig. 4B). Transcriptional regulation of C/EBP homologous protein (Ddit3, also known as Chop) occurs by these transcription factors and was increased nearly 5-fold in HSVLF-fed SCD1 LKO mice relative to Lox (Fig. 4C). Other genes indicative of ER stress and UPR including Hspa5 (Grp78), Asns, Atf3, PP1r15a (Gadd34), and Hsp90b1 (Grp94) were also induced in SCD1 LKO mice (Fig. 4C, D). Despite the substantial ER stress induced by HSVLF in SCD1 LKO mice, dietary oleate supplementation completely normalized several key ER stress markers in SCD1 LKO mice (Fig. 4A, C), whereas stearate supplementation did not rescue ER stress genes (Fig. 4C). We also assessed hepatic expression of several ER stress genes after feeding Lox and LKO mice the lipogenic high-sucrose HC diet for 10 days. Expression of Atf3, Asns and Ddit3 were all significantly upregulated in LKO mice while expression of other ER stress genes, including Pp1r15a and spliced Xbp-1, were increased but the effect was attenuated and did not reach significance (Fig. 4E). These data suggest that the reduced capacity to specifically increase hepatic MUFA synthesis during lipogenic, dietary conditions leads to the development of hepatic ER stress in SCD1 LKO mice. 
We also examined the integrity and function of hepatic mitochondria of HSVLF-fed SCD1 LKO mice. There were no differences in mitochondrial membrane potential, membrane fluidity or dehydrogenase activity in SCD1 LKO mice compared to Lox (Fig. 4F). Furthermore, mitochondrial ultrastructure showed comparable morphology between SCD1 LKO and Lox mice (Fig. 4G). To determine if altered composition of membrane phospholipids may explain the stress phenotype, we measured total phosphatidylcholine (PC) and total phosphatidylethanolamine (PE), two major membrane phospholipids in hepatic microsomes and mitochondria. There were no differences between LKO and Lox HSVLF-fed mice in either phospholipid or in the PC/PE ratio in microsomes and mitochondria (Supplementary Fig. 5). Mitochondrial reactive oxygen species (ROS) were measured to determine if they contributed to the development of liver ER stress and inflammation in LKO mice. The production of ROS in liver mitochondria were significantly reduced in HSVLF-fed LKO mice compared to Lox counterparts (Supplementary Fig. 6). Collectively, these data indicate that limited hepatic MUFA is associated with disruption of ER function without damage to mitochondria.

HSVLF-induced liver stress and inflammation is reduced by de novo hepatic oleate, but not palmitoleate, synthesis in SCD1 GKO mice [2]

Next, we questioned whether increased local hepatic de novo oleate or palmitoleate synthesis, the major products of the SCD1-catalyzed reaction, in GKO mice would prevent HSVLF-induced liver stress and inflammation. We generated two liver-specific transgenic mouse models: one expressing human SCD5 (synthesizes oleate) and one expressing mouse $S c d 3$ (synthesizes palmitoleate) and crossed these 
transgenes into the SCD1 GKO background to generate Scd $1^{-1}$; SCD5 transgenic $(\mathrm{Tg})$ mice (GLS5) and Scd1 ${ }^{-;}$; Scd3 Tg mice (GLS3) (19). In mice, under lipogenic dietary conditions Scd1 is the predominant isoform expressed in liver; Scd3 expression is largely limited to skin and the Harderian and preputial glands (24-26).

The use of liver-specific transgenic desaturase mouse models fed the HSVLF diet allowed us to assess the effects specifically of local, liver-derived MUFA on hepatic function. We measured oleate and palmitoleate levels in the major hepatic lipid classes, including TG, CE, and PL (Fig. 5A-C). Although MUFA were not restored to WT levels, 18:1n-9 and 16:1n-7 were increased significantly in GLS5 and GLS3, respectively, relative to GKO mice in TG and PL fractions. In the CE class 18:1n-9 was elevated in GLS5 mice, but $16: 1 n-7$ was not significantly increased in GLS3 compared to GKO mice. The low body weight and adiposity phenotypes of HSVLF-fed GKO mice were completely restored to WT levels in GLS5 mice but not GLS3 mice (Supplementary Table 1). Total liver weight was significantly greater in GKO, GLS5 and GLS3 mice compared to WT (Fig. 5D) but liver TG analysis indicated that the increased weight was not explained by lipid accumulation (Supplementary Table 1). As previously reported, GKO mice are protected from high-fat and high-carbohydrate diet-induced hepatic lipid accumulation and did not accumulate liver TG in the current study. Liver TG were modestly increased in GLS5 mice relative to GKO but not changed in GLS3 mice (Supplementary Table 1).

Consistent with HSVLF-fed SCD1 LKO mice, plasma ALT activity was significantly elevated in GKO mice relative to WT but significantly reduced in GLS5 mice (Fig. 5E). ALT activity was also reduced in GLS3 mice but the reduction was not significantly 
different from the GKO group and remained significantly higher than WT (Fig. 5E). We then assessed expression of hepatic inflammation genes. Tnfa, Emr1, Icam1 and Cd68 were generally upregulated in GKO and GLS3 mice (Fig. 5F, H) but normalized to WT levels in GLS5 mice (Fig. 5F). Expression of ER stress genes showed a similar pattern with Atf3, Asns, Hspa5, and Ddit3 significantly increased in GKO and GLS3 mice but normalized in GLS5 mice to WT levels (Fig. 5G, I). These results suggest that de novo synthesized oleate can resolve hepatic ER stress and inflammatory pathways when exogenous dietary MUFA are limiting but palmitoleate has little effect. The GLS5 transgenic model further confirms the stearate- and oleate supplementation feeding study results (Fig. 3,4 ) in the SCD1 LKO model and clearly demonstrates a role for hepatic oleate availability in preventing the development of lipogenic diet-induced liver inflammation and ER stress.

\section{Liver-specific deletion of PGC-1a reduces expression of inflammation and ER stress response genes in SCD1 LKO mice [2]}

Our past work revealed that both liver-specific and global SCD1 deficiency induces upregulation of hepatic Ppargc1a (PGC-1a) expression under HSVLF dietary conditions $(17,18)$. In HSVLF-fed male mice, hepatic Ppargc1a expression is reduced in GLS5 mice but not significantly lowered in GLS3 mice (Fig. 6A). This suggests that oleate negatively influences PGC-1a expression but palmitoleate has less of an effect. To determine if PGC-1a mediates the liver ER stress response in SCD1 LKO mice, we generated double liver knockout mice deficient in both Scd1 and Ppargc1a expression in liver (DLKO) (Fig. 6B, C). We then fed Lox, PGC-1a LKO, SCD1 LKO and DLKO mice the HSVLF diet for a period of 14 days and measured markers of ER stress and 
inflammation. Plasma ALT was elevated in DLKO mice to the same level as SCD1 LKO mice and was significantly higher in both compared to Lox (Fig. 6D). The effect of hepatic PGC-1a deletion in SCD1 LKO mice on expression of inflammation genes in the liver was generally modest. The expression of Tnfa, Emr1 and Icam1 were partially restored in DLKO mice, to a level not significantly different from Lox (Fig. 6E). The expression of Cd68 remained elevated in DLKO compared to Lox (Fig. 6E).

The deletion of PGC-1a in SCD1 LKO mice had a dramatic impact on the expression of hepatic ER stress response genes, where the increased expression observed in SCD1 LKO was broadly blunted in DLKO mice. Specifically, the spliced isoform of Xbp1, Ddit3, Hspa5, Atf3, Asns, Hsp90b1, and Ppp1r15a expression were significantly reduced in DLKO mice compared to SCD1 LKO mice and not significantly different from Lox (Fig. 6F, G). Although ER stress was alleviated in DLKO mice, the decreased body weight elicited by HSVLF feeding in male and female LKO mice was not affected by deletion of PGC-1 $\alpha$ in DLKO mice (Supplementary Table 2, 3). These data suggest that the increase in PGC-1 $\alpha$ expression during SCD1 deficiency may at least partially mediate the HSVLF-induced hepatic ER stress and inflammation but not the decreased body weight, thus decoupling the ER stress from metabolic phenotypes of SCD1 LKO mice. 


\section{Discussion [1]}

Consumption of high-carbohydrate diets, especially those high in sugar, contributes to the development of hepatic steatosis in part through stimulation of hepatic de novo lipogenesis. The major products of de novo lipogenesis include SFA and MUFA, which serve as substrates for the synthesis of cellular lipids critical for liver homeostasis. We utilized several mouse models to manipulate hepatic MUFA content, including SCD1 LKO mice fed HSVLF and HC diets and liver transgenic human SCD5 or mouse Scd3 mice, which allowed us to restrict and restore MUFA supply and de novo synthesis selectively in liver. We reveal that oleate, the major MUFA species, is both necessary and sufficient to prevent hepatic dysfunction, ER stress and inflammation during high-carbohydrate feeding. We further identified PGC-1 $\alpha$ as a potential mediator of the liver ER stress response in SCD1-deficient mice.

SCD1 LKO mice exhibit a high level of hepatic stress and inflammation. Liver injury is likely initiated from hepatocytes as the reduction in SCD1 expression in LKO mice were generated by use of the albumin promoter. However, damaged hepatocytes provoke the stress response in proinflammatory cells, which results in hepatic inflammatory infiltration. Thus, liver injury in LKO mice is likely derived from both parenchymal and non-parenchymal cells. The causal relationship between reduced hepatic MUFA and liver stress during lipogenic dietary conditions was demonstrated through fatty acid supplementation to the HSVLF diet in which the addition of oleate completely reversed the liver stress and inflammation in SCD1 LKO mice, while supplementation of stearate did not have an effect. Indeed, SFA are generally considered to exert lipotoxic effects that stimulate inflammatory and ER stress 
responses (4-7). In this study, dietary stearate supplementation did not exacerbate the hepatic stress in LKO mice, which may be explained by the already heightened level of stress induced by the high-carbohydrate diet in SCD1 LKO mice.

The indispensable role of hepatic MUFA in maintaining liver homeostasis was further demonstrated in the SCD1 LKO model, as other major extrahepatic SCD1expressing tissues, such as adipose, did not rescue liver dysfunction even with intact SCD1 expression. Our data show that white adipose tissue TG oleate concentrations were not altered in HSVLF-fed LKO mice, suggesting that adipose tissue was not able to compensate the limited MUFA availability in liver. As adipose tissue is a major tissue contributing to the circulating FFA pool, its inability to rescue hepatic MUFA restriction was further revealed by the finding that nonesterified oleate in circulating FFA fraction was significantly reduced in LKO mice. Together these data indicate that during highcarbohydrate feeding, sufficient hepatic MUFA availability, either through exogenous dietary supply or through local endogenous synthesis, is required for normal hepatic function, and the reduction of MUFA in this tissue is not compensated by SCD1 activity in extrahepatic tissues.

To confirm the role and specificity of hepatic MUFA in liver function, we employed a gain-of-function strategy by generating two transgenic mouse models with liver-specific transgenic expression of human SCD5 and mouse Scd3 in global SCD1 deficient mice. These models exhibit elevated $\Delta 9$-desaturase activity selectively in the liver, and generate oleate and palmitoleate, respectively (19). These transgenic models allowed us to differentiate the liver protective actions of oleate and palmitoleate, the two major products of SCD-catalyzed reactions. We found that oleate, but not palmitoleate, 
specifically plays a role in maintenance of hepatic function, such that hepatic oleate is both necessary and sufficient to prevent high-carbohydrate-induced liver stress. This is in accordance with a report in $\mathrm{C} 2 \mathrm{C} 12$ myotubes that oleate rescues the detrimental effects of palmitate, which includes increases in diacylglycerol, activation of the NF-KB pathway and subsequent inflammation and insulin resistance (27).

PGC-1 $\alpha$ is a transcriptional coactivator that exerts regulation over a number of metabolic pathways in several tissues and is particularly important in mediating the metabolic switch from the fed to fasted state (28). PGC-1a has also been shown to be essential for the adaptive response to exercise in skeletal muscle, where it coactivates ATF6 $\alpha$ to induce upregulation of ER stress genes and the UPR program (9). Upregulation of hepatic PGC-1a and ER stress genes is observed in SCD1-deficient mice upon high-carbohydrate feeding, and we questioned whether PGC-1a mediates the connection between hepatic oleate and hepatic ER stress. Indeed, as demonstrated in this study, SCD1 LKO mice lacking PGC-1 $\alpha$ exhibit dramatically reduced expression of ER stress related genes. This suggests that increased levels of hepatic oleate may prevent high-carbohydrate diet-induced ER stress through down-regulation of PGC-1a. Fatty acid mediated regulation of PGC-1a gene expression was previously demonstrated in which treatment of cultured primary hepatocytes with individual free fatty acid species revealed a significant upregulation of PGC-1 $\alpha$ mRNA expression upon stearate treatment but no induction with oleate (29). Other work in primary human myocytes demonstrated that oleate, palmitate or TNF- $\alpha$ treatment can all individually induce hypermethylation of the PGC-1 $1 \alpha$ promoter, suggesting a possible mechanism through which fatty acids can directly regulate expression of this transcriptional 
coactivator (30). However, the mechanism through which oleate represses PGC-1a expression in SCD1-deficient mice fed a high-carbohydrate diet remains unknown. In contrast to the effect on ER stress markers, liver-specific deficiency of PGC-1 $\alpha$ did not influence plasma ALT activity and it only modestly influenced the expression of hepatic inflammation genes, thus implicating other regulators or pathways involved in the HSVLF-induced liver injury and inflammation phenotypes in SCD1-deficient mice.

Our previous work in both GKO and LKO mice demonstrated that hepatic SCD1 deficiency attenuates the high-carbohydrate diet-induced adiposity, hepatic steatosis and stimulation of the de novo lipogenesis program $(17,18)$. However, our current and previous work indicates that the protection from high-carbohydrate diet-induced adiposity and liver steatosis co-exists with liver ER stress and inflammation in these mice. This is surprising because ER stress and inflammation are associated with and commonly implicated as causative factors in development of metabolic disorders including increased adiposity and hepatic steatosis. Thus, hepatic SCD1 might serve as a metabolic link between liver stress response and hepatic steatosis, and lack of hepatic SCD1 may uncouple liver stress and inflammation from development of other detrimental metabolic disturbances. Furthermore, despite hepatic stress and inflammation, blood glucose levels do not become elevated in SCD1 LKO mice (18). In the current study, plasma glucose was significantly lower upon insulin injection. While this may suggest improved peripheral insulin sensitivity, it might alternatively reflect the block in the hepatic gluconeogenic program previously described in LKO mice (18).

In summary, this study clearly reveals that maintenance of hepatic oleate, either through dietary sources or de novo synthesis, is indispensable in the maintenance of 
normal liver function. The inability of palmitoleate to prevent liver ER stress and inflammation suggests differential cellular roles for the SCD1 products oleate and palmitoleate in liver homeostasis. Furthermore, we show that hepatic oleate levels influence PGC-1 $\alpha$ expression and that PGC-1 $\alpha$ potentiates the ER stress response in liver. Future work will be needed to uncover the molecular mechanism of how oleate regulates PGC-1 $\alpha$ and the downstream pathways that lead to ER stress under certain dietary conditions such as high-carbohydrate low-fat diets.

\section{Financial support [1]}

This work was supported by NIH R01 DK062388, ADA 7-13-BS-118 and USDA Hatch W2005 (to J.M.N.). X.L. was supported by American Heart Association Postdoctoral Fellowship. M.S.B. was supported by T32 DK 007665 and NCI R25 CA094880. M.T.F was supported by NIH postdoctoral training grant T32 DK 007665 and an American Heart Association postdoctoral fellowship.

\section{Author contributions [1]}

X.L., M.S.B., M.T.F. and J.M.N. were involved in study design and data interpretation. X.L., M.S.B., and M.T.F. conducted experim ents and data analysis. X.L., M.S.B. and J.M.N. wrote the manuscript. 


\section{References}

1. Magkos F, Yannakoulia M, Chan JL, Mantzoros CS. Management of the metabolic syndrome and type 2 diabetes through lifestyle modification. Ann Rev Nutr. 2009;29:223-256.

2. Vos MB, Lavine JE. Dietary fructose in nonalcoholic fatty liver disease. Hepatology. 2013;57:2525-2531.

3. Lumeng CN, Saltiel AR. Inflammatory links between obesity and metabolic disease. J Clin Invest. 2011;121:2111-2117.

4. Malhi H, Kaufman RJ. Endoplasmic reticulum stress in liver disease. J Hepatol. 2011:54:795-809.

5. Solinas G, Naugler W, Galimi F, Lee M-S, Karin M. Saturated fatty acids inhibit induction of insulin gene transcription by JNK-mediated phosphorylation of insulin-receptor substrates. Proc Natl Acad Sci USA. 2006;103(44):16454-16459.

6. Holzer RG, Park E-J, Li N, Tran H, Chen M, Choi C, et al. Saturated fatty acids induce c-Src clustering within membrane subdomains leading to JNK activation. Cell 2011;147:173-184. 
7. Leamy AK, Egnatchik RA, Young JD. Molecular mechanisms and the role of saturated fatty acids in the progression of non-alcoholic fatty liver disease. Prog Lipid Res. 2013;52:165-174.

8. Ozcan U, Cao Q, Yilmaz E, Lee AH, Iwakoshi NN, Ozdelen E, et al. Endoplasmic reticulum stress links obesity, insulin action, and type 2 diabetes. Science. 2004;306:457-461.

9. Wu J, Ruas JL, Estall JL, Rasbach KA, Choi JH, Ye L, et al. The unfolded protein response mediates adaptation to exercise in skeletal muscle through a PGC1 $\alpha /$ ATF6 $\alpha$ complex. Cell Metab. 2011;13:160-169.

10. Ntambi JM, Miyazaki M. Recent insights into stearoyl-CoA desaturase-1. Curr Opin Lipidol. 2003;14:255-261.

11. Ntambi JM, Miyazaki M. Regulation of stearoyl-CoA desaturases and role in metabolism. Prog Lipid Res. 2004;43:91-104.

12. DeWille JW, Farmer SJ. Postnatal dietary fat influences mRNAS involved in myelination. Dev Neurosci. 1992;14:61-68. 
13. Kim JH, Kim Y, Lee SD, Lopez I, Arnold RS, Lambeth JD, et al. Selective activation of phospholipase D2 by unsaturated fatty acid. FEBS Lett. 1999;454:42-46.

14. Ntambi JM. Regulation of stearoyl-CoA desaturase by polyunsaturated fatty acids and cholesterol. J Lipid Res. 1999;40:1549-1558.

15. Neuschwander-Tetri BA. Hepatic lipotoxicity and the pathogenesis of nonalcoholic steatohepatitis: the central role of nontriglyceride fatty acid metabolites. Hepatology. 2010;52:774-788.

16. Gentile CL, Frye MA, Pagliassotti MJ. Fatty acids and the endoplasmic reticulum in nonalcoholic fatty liver disease. Biofactors. 2011;37(1):8-16.

17. Flowers MT, Keller MP, Choi Y, Lan H, Kendziorski C, Ntambi JM, et al. Liver gene expression analysis reveals endoplasmic reticulum stress and metabolic dysfunction in SCD1-deficient mice fed a very low-fat diet. Physiol Genomics 2008;33:361-372.

18. Miyazaki M, Flowers MT, Sampath H, Chu K, Otzelberger C, Liu X, et al. Hepatic stearoyl-CoA desaturase-1 deficiency protects mice from carbohydrateinduced adiposity and hepatic steatosis. Cell Metab. 2007;6:484-496. 
19. Burhans MS, Flowers MT, Harrington KR, Bond LM, Guo CA, Anderson RM, Ntambi JM. Hepatic oleate regulates adipose tissue lipogenesis and fatty acid oxidation. J Lipid Res. 2015 [Epub ahead of print].

20. Miyazaki M, Dobrzyn A, Man WC, Chu K, Sampath H, Kim H-J, et al. StearoylCoA desaturase 1 gene expression is necessary for fructose-mediated induction of lipogenic gene expression by sterol regulatory element-binding protein-1cdependent and independent mechanisms. J Biol Chem 2004;279:25164-25171.

21. Malhi H, Gores GJ. Molecular mechanisms of lipotoxicity in nonalcoholic fatty liver disease. Semin Liver Dis. 2008;28:360-369.

22. Giorgi C, De Stefani D, Bononi A, Rizzuto R, Pinton P. Structural and functional link between the mitochondrial network and the endoplasmic reticulum. Int $\mathrm{J}$ Biochem Cell Biol. 2009;41:1817-1827.

23. Minamino T, Kitakaze M. ER stress in cardiovascular disease. J Mol Cell Cardiol. 2010;48:1105-1110.

24. Zheng Y, Prouty SM, Harmon A, Sundberg JP, Stenn KS, Parimoo S. Scd3-a novel gene of the stearoyl-CoA desaturase family with restricted expression in skin. Genomics. 2001;71:182-191. 
25. Miyazaki M, Kim HJ, Man WC, and Ntambi JM. Oleoyl-CoA is the major de novo product of stearoyl-CoA desaturase 1 gene isoform and substrate for the biosynthesis of the Harderian gland 1-alkyl-2,3-diacylglycerol. J. Biol. Chem. 2001;276:39455-39461.

26. Miyazaki M, Gomez FE, Ntambi JM. Lack of stearoyl-CoA desaturase-1 function induces a palmitoyl-CoA Delta6 desaturase and represses the stearoyl-CoA desaturase-3 gene in the preputial glands of the mouse. J. Lipid Res. 2002;43:2146-2154.

27. Coll T, Eyre E, Rodriguez-Calvo R, Palomer X, Sanchez RM, Merlos M, et al. Oleate reverses palmitate-induced insulin resistance and inflammation in skeletal muscle cells. J Biol Chem. 2008;283:11107-11116.

28. Lin J, Handschin C, Spiegelman BM. Metabolic control through the PGC-1 family of transcription coactivators. Cell Metab. 2005;1:361-370.

29. Lin J, Yang R, Tarr PT, Wu P-H, Handschin C, Li S, et al. Hyperlipidemic effects of dietary saturated fats mediated through PGC-1 $\beta$ coactivation of SREBP. Cell. 2005;120:261-273. 
30. Barres R, Osler ME, Yan J, Rune A, Fritz T, Caidahl K, et al. Non-CpG methylation of the PGC-1 $\alpha$ promoter through DNMT3B controls mitochondrial density. Cell Metab. 2009;10:189-198.

Fig. 1. Monounsaturated fatty acids are reduced in hepatic lipids and plasma FFA of LKO mice. Lox and LKO mice were fed a HSVLF diet for 10 days. Fatty acid composition of liver and plasma lipids was determined by gas liquid chromatography ( $\mathrm{n}$ = 6/group). (A) TG, (B) FFA and (C) CE fractions in liver tissue and (D) FFA in plasma were analyzed. Values are mean \pm SEM, ${ }^{*} p<0.05$ vs. Lox counterparts by Student's two-tailed $t$ test.

Fig. 2. White adipose tissue (WAT) fatty acid composition and lipogenic gene expression is not altered in LKO mice. Lox and LKO mice were fed HSVLF diet for 10 days. (A) Fatty acid analysis of the TG fraction in epididymal WAT depot was conducted by gas liquid chromatography ( $n=4-6 /$ group). (B) Deficiency of SCD1 in liver does not influence relative fatty acid synthesis gene expression including Srebf1, Acaca, Fasn and Scd1 ( $\mathrm{n}=5$-8/group). (C) Immunoblot analysis of SCD1 protein in whole tissue lysate of the epididymal WAT depot. Values are mean \pm SEM.

Fig. 3. High-sucrose lipogenic diets induces hepatic inflammation in LKO mice. 
(A-E) Hepatic injury and inflammation were assessed in chow, 10-day HSVLF, 10-day HSVLF + 18:1n-9 and 10-day HSVLF + 18:0-fed Lox and LKO mice. (A) Plasma ALT activity ( $n=3-6 /$ group) and (B) necrotic foci in liver tissue sections, (C and D) Relative hepatic expression levels of proinflammatory genes. (E) The ratio of wet to dry liver tissue weight in HSVLF-fed Lox and LKO mice. (F) Lox and LKO mice were fed a lipogenic high-carbohydrate $(\mathrm{HC}, 12 \%$ fat $)$ diet for 10 days and relative hepatic expression of proinflammatory genes was measured ( $n=5-6 /$ group). ( $G$ and $H$ ) Lox and LKO mice were fed a chow or HSVLF diet for 10 weeks ( $n=5-7 / g r o u p) ;(G)$ plasma ALT activity and $(\mathrm{H})$ necrotic foci in liver tissue were assessed. Values are mean \pm SEM, ${ }^{*} p$ $<0.05$ vs. Lox counterparts by Student's two-tailed $t$ test.

Fig. 4. Hepatic ER stress is induced in LKO mice fed HSVLF diet. (A-D) Lox and LKO mice were fed a chow, HSVLF, HSVLF $+18: 1 \mathrm{n}-9$, or HSVLF $+18: 0$ diet for 10 days and ER stress was assessed. (A) Immunoblot analysis was used to determine phosphorylated elF2 $\alpha$ and total elF2 $\alpha$ protein in whole liver tissue lysates. (B and C) Relative mRNA expression levels were determined for ER stress response genes $(n=$ 5-6/group) including (B) total and spliced Xbp-1, (C) Ddit3, Hspa5, Asns and Atf3, (D) Ppp1r15a and Hsp90b1. (E) Lox and LKO mice were fed a lipogenic high-carbohydrate ( $\mathrm{HC}, 12 \%$ fat) diet for 10 days and relative hepatic expression of ER stress genes was measured ( $n=5-6 /$ group). (F) Mitochondria were isolated from fresh liver tissue of Lox and LKO mice fed the HSVLF diet for 10 days ( $n=4-6 / g r o u p$ ). The inner/outer mitochondria membrane potential, mitochondrial membrane fluidity and activity of mitochondria dehydrogenase were assessed. (G) Electron microscopy of mitochondrial 
ultra-structure. Values are mean $\pm \mathrm{SEM},{ }^{*} p<0.05$ vs. HSVLF-fed Lox mice by Student's two-tailed $t$ test.

Fig. 5. Markers of hepatic stress and inflammation are normalized by hepatic SCD5 expression. WT, GKO, GLS5 and GLS3 female mice were fed HSVLF for 10 days $(n=6-10 /$ group). (A) Hepatic oleate $(18: 1 n-9)$ and palmitoleate $(16: 1 n-7)$ were measured in liver TG by gas liquid chromatography. (B) Total liver tissue weight. (C) Plasma ALT activity. (D-G) Relative expression levels of inflammation and ER stressrelated genes were measured in WT, GKO, GLS5 and GLS3. Values are mean \pm SEM, ${ }^{*} p<0.05$ vs. WT, ${ }^{\#} p<0.05$ vs. GKO; one-way ANOVA analysis.

Fig. 6. Hepatic PGC-1a deficiency reduces hepatic ER stress gene expression in SCD1 LKO mice. (A) WT, GKO, GLS5 and GLS3 mice were fed a HSVLF diet for 10 days and relative hepatic expression of PGC-1 $\alpha$ was measured $(n=4-6 / g r o u p)$. (B and C) Lox, PGC-1a LKO, SCD1 LKO or DLKO mice were fed a HSVLF diet for 14 days. Mice were $4 \mathrm{~h}$ fasted prior to tissue collection ( $n=12-14 /$ group). Relative hepatic gene expression levels of (B) Scd1 and (C) Ppargc1a. (D) Plasma ALT activity after 14 days of HSVLF diet. (E) Hepatic expression of inflammatory markers and ( $F$ and $G$ ) ER stress genes in mice as described in B and C. Values are mean \pm SEM, ${ }^{*} p<0.05$ vs. Lox or vs. WT, ${ }^{\prime} p<0.05$ vs. GKO or SCD1 LKO; one-way ANOVA analysis. 
A

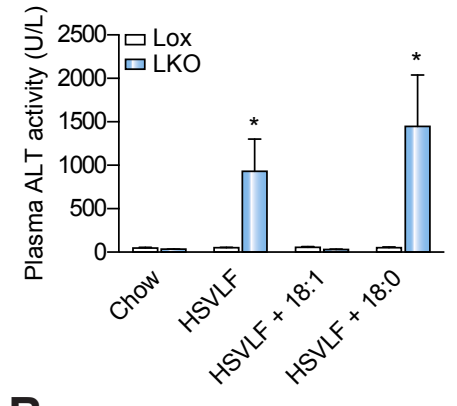

B

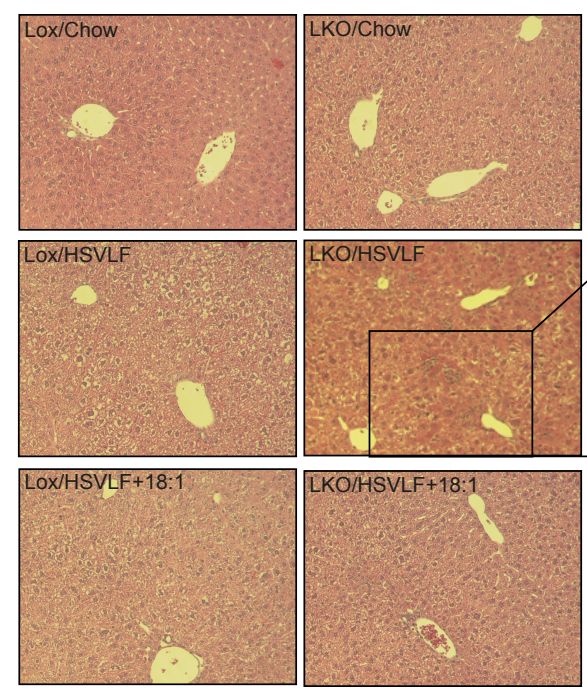

Liver Icam1

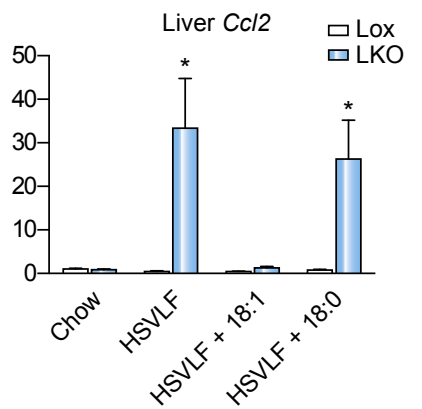

LKO/HSVLF
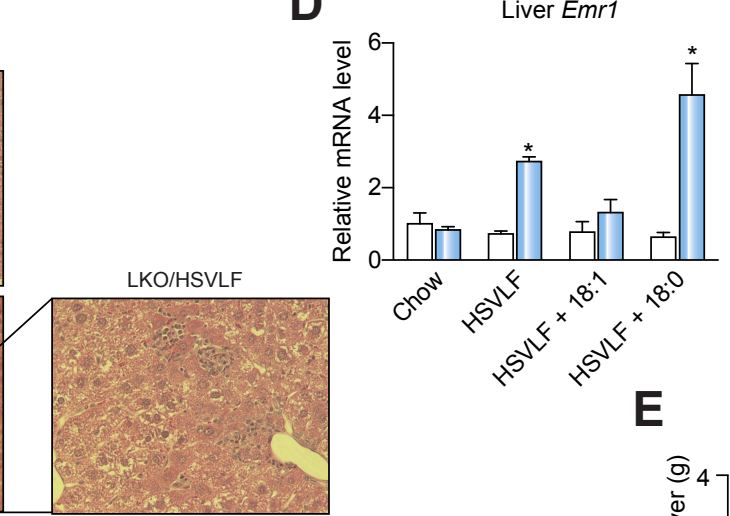

$\begin{array}{ll}\text { Liver Cd68 } & \square \text { Lox } \\ & \square \text { LKO }\end{array}$
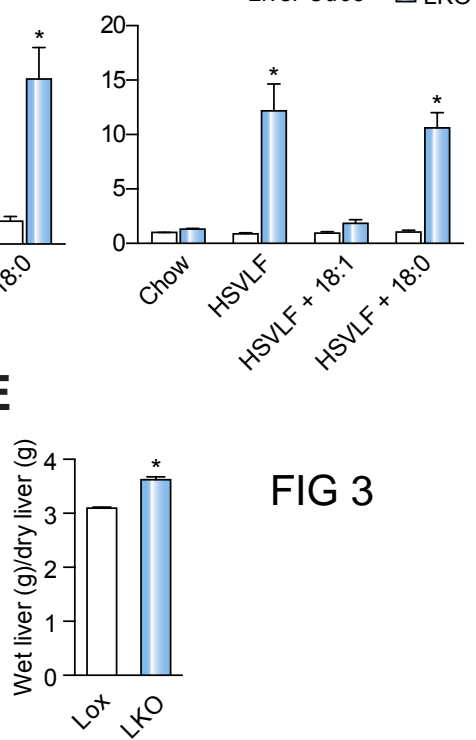

FIG 3

H

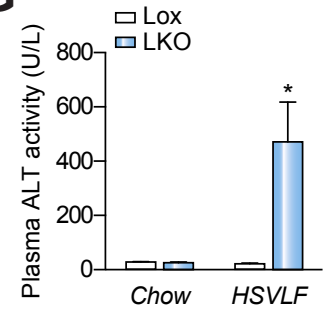

Lox HSVLF

LKO HSVLF

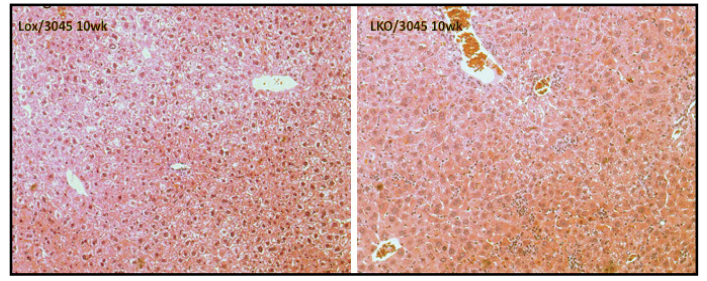


A

Triglycerides
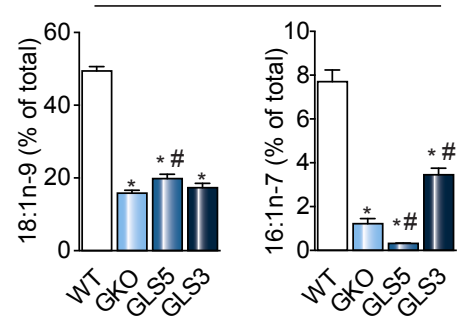

E

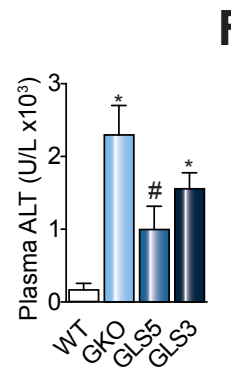

F

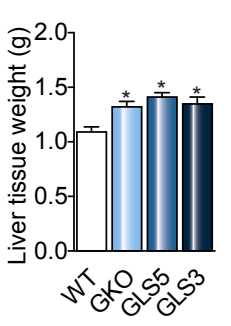

H
B
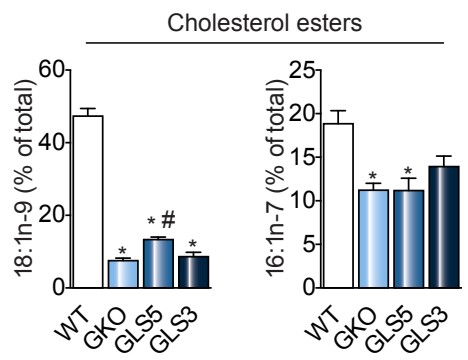

C

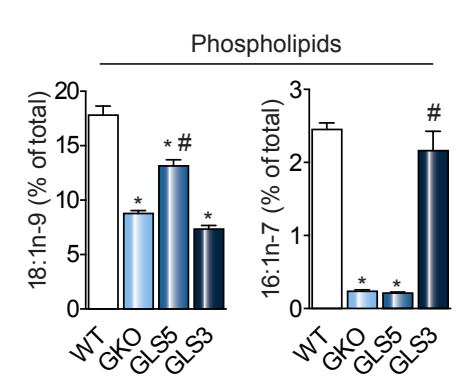

G

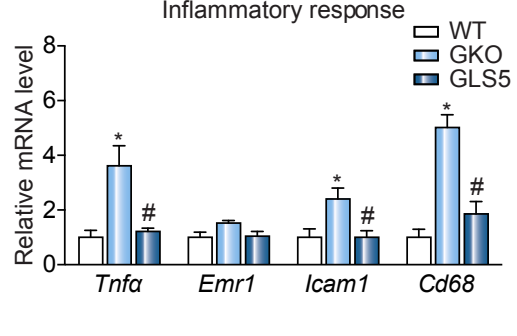

ER stress response

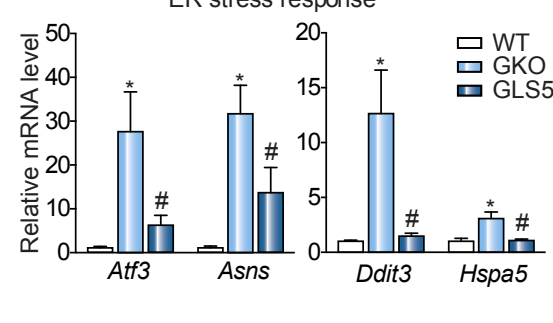

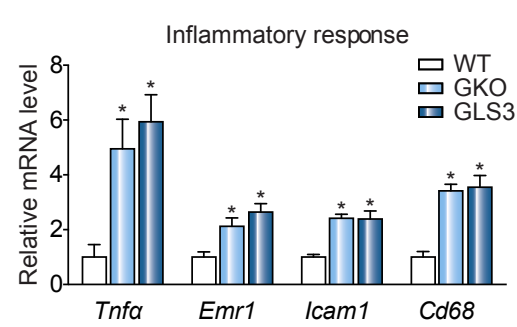

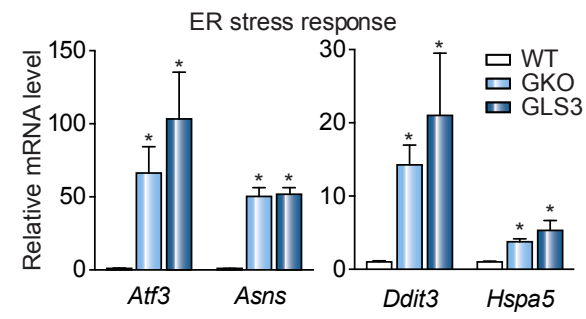

FIG 5 


\title{
High-carbohydrate
}

\author{
low-fat diet
}
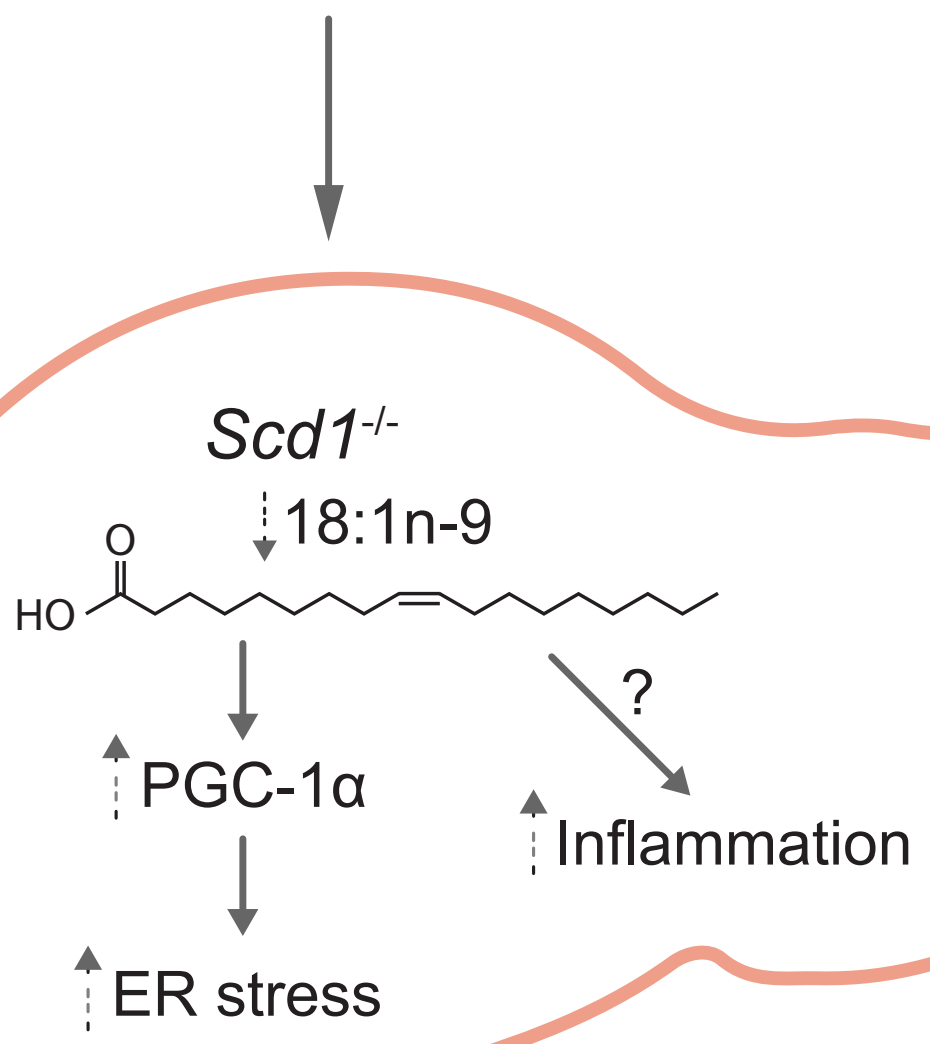